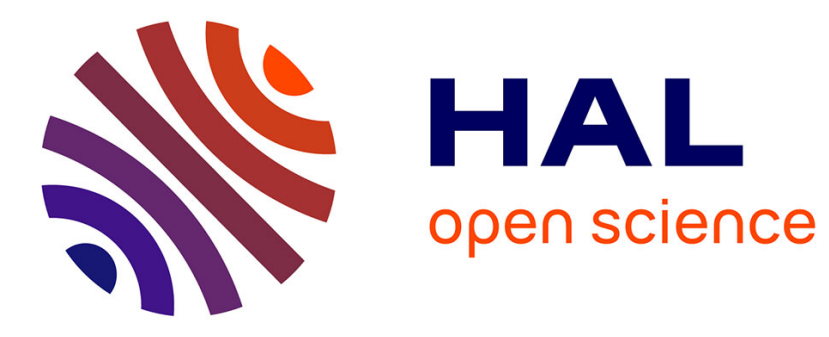

\title{
A biobjective formulation for filming sport events problem using drones
}

Luigi Di Puglia Pugliese, Francesca Guerriero, Enrico Natalizio, Nicola Roberto Zema

\section{- To cite this version:}

Luigi Di Puglia Pugliese, Francesca Guerriero, Enrico Natalizio, Nicola Roberto Zema. A biobjective formulation for filming sport events problem using drones. 9th IEEE International Conference on Intelligent Data Acquisition and Advanced Computing Systems: Technology and Applications (IDAACS), IEEE, Sep 2017, Bucharest, Romania. pp.639-644, 10.1109/IDAACS.2017.8095170 . hal-01672186

\section{HAL Id: hal-01672186 https://hal.science/hal-01672186}

Submitted on 23 Dec 2017

HAL is a multi-disciplinary open access archive for the deposit and dissemination of scientific research documents, whether they are published or not. The documents may come from teaching and research institutions in France or abroad, or from public or private research centers.
L'archive ouverte pluridisciplinaire HAL, est destinée au dépôt et à la diffusion de documents scientifiques de niveau recherche, publiés ou non, émanant des établissements d'enseignement et de recherche français ou étrangers, des laboratoires publics ou privés. 


\title{
A biobjective formulation for filming sport events problem using drones
}

\author{
Luigi Di Puglia Pugliese ${ }^{1}$, Francesca Guerriero ${ }^{1}$, Enrico Natalizio ${ }^{2}$, Nicola Roberto Zema ${ }^{2}$ \\ ${ }^{1}$ Department of Mechanical, Energy and Management Engineering, University of Calabria, 87036, Rende. \\ e-mail: \{luigi.dipugliapugliese,francesca.guerriero\}@unical.it \\ 2 Lab. Heudiasyc, UMR CNRS 7253, France. e-mail: \{enrico.natalizio,nicola.zema\}@hds.utc.fr
}

\begin{abstract}
In this paper we consider the problem of filming sport events, ensuring high quality image transmission to the spectators. A fleet of drones is used to cover the area in which the play takes place and to track all events. Auxiliary drones are used to support filming drones in order to transmit high quality image to the disadvantaged spectators with respect to the event that takes place in a certain instant of time. The main goal is to decide the position of the filming and auxiliary drones, aiming to provide high view satisfaction and, at the same time, minimizing the distance travelled by the drones. The two objectives are in conflict with each other, thus we formulate the problem as a bi-objective mixed integer model. A unique optimal solution does not exist, rather a set of optimal solutions, named Pareto optimal, can be considered efficient.
\end{abstract}

Keywords - sport event film problem, mobile camera drones, drones routing problem, multi-objective optimization

\section{INTRODUCTION}

In this paper, we address the problem of managing a fleet of drones for filming sport events and streaming images to the spectators within the stadium.

The Sport Event Filming (SEF) problem, recently introduced in [1], aims at determining a planning of the filming drones in terms of positions they have to occupy and the time in which the drones should be in each position. The objective is to film each event maximizing the viewer satisfaction of the spectators. In order to have the maximum satisfaction, each event have to be monitored for all its duration. In the case the drone is late with respect to the event, the viewer satisfaction decrease reaching the value of zero if the event is not filmed. In addition, we suppose there are parts of the stadium, i.e. the corners, for which the quality of images sent to the spectators, located in the opposite ones, decreases. To overcome this issue, we use a fleet of supporting drones that operate as a relay between the filming drone and the disadvantaged spectators.

The aim of this paper is to define an optimization model that gives insight on how to manage the fleet of filming and supporting drones when the play takes place. In particular, we suppose to have a-priori knowledge of the events in term of positions in the stadium and time in which they happen. This is an off-line configuration that provides to the decision maker an initial planning of the fleets and suggests the number, the positions and the movements of both filming and supporting drones.

From a modeling viewpoint, the problem belongs to the Vehicle Routing Problem with Time Windows (VRPTW) family, where vehicles, starting from a depot, visit a set of customers within their time window. For more details on the VRPTW and its variants, the reader is referred to [2]. Each event can be viewed as a customer and the time between the end of the event and its starting time represents the time window. All events have to be visit by the drones within their time windows. We admit the drone can start to monitor the position in which an event will take place before it start.

Since the viewer satisfaction is related to the time in which the drone starts to film the event, the time windows are considered soft in the sense that the quality of the service is influenced by the instant time in which the customer (event in our application) is visited. More details on the VRP with soft time windows can be found in [3][5].

In this paper we formulate the SEF problem with supporting drones (SEF-SD) as a variant of the VRPTW, where two different fleets of vehicles have to be manage in order to film the events within their time windows and provide high quality image using supporting drones.

The distance travelled by the drones has to be taken into consideration. Indeed, assuming a constant flying speed and altitude, the distance travelled is related to the flying autonomy of the drone. Thus, this parameter needs to be controlled and it plays a crucial role in the determination of the optimal planning.

Whilst the viewer satisfaction has to be maximized in order to ensure quality of service requirements, the distance travelled needs to be minimized in order to preserve the efficiency of the system in term of energy consumption. The two objectives are in conflict with each other. Indeed, to guarantee a higher viewer satisfaction, the drones have to move frequently in order to film the events and supporting drones have to be activated.

We present the SEF-SD under the bi-objective opti- 
mization framework, where a unique optimal solution does not exist. Rather, a set of solutions, named Pareto, can be considered optimal in the sense each of them optimizes the two objective functions.

The common way to address bi-objective optimization problem is to define a scalarization function, that allows to manage the problem as a single-objective one. Modifying the parameters of the scalarizing function, different Pareto optimal solutions can be found.

In this paper, we consider two types of scalarization functions. The first one relies on a convex combination of the objective functions, and the second uses the Chebishev-norm as in the reference point methodology [6], [7]. In addition, we apply the so-called $\epsilon$-constraint method [8], where an objective function is optimized and the other is viewed as a hard constraint. Varying the right-hand side of the constraint, different Pareto optimal solutions can be determined.

The paper is organized as follows. Section II formalizes the viewer satisfaction and the problem. The bi-objective formulation is given along with the methods adopted to search Pareto optimal solutions. Section III presents an illustrative example with the aim of showing how the proposed solution strategies behaves. Section IV concludes the paper. The mathematical formulation of the problem is presented in the appendix.

\section{PRoblem DEFINITION AND SOLUTiON APPROACHES}

In this section, we describe the problem at hand, along with its mathematical formulation as a bi-objective model. Then, we propose three solution strategies in order to obtain Pareto solutions.

a) Problem definition: We assume that the stadium is characterized by two typologies of zones. The first one, located in the center, and the others are represented by the four corners. According to the measurements presented in [9], we define as 1-Hop the center of the field, i.e. the first zone typology.

The filming drones broadcast high definition images evaluated as $S_{\max H}$ of the events in the 1-Hop area without the help of the supporting drones. On the other hand, the images sent from the corners are of low definition $\left(S_{\max L}\right)$ for the spectators located at the opposite corner. In this situation, the supporting drones are used as relay between filming drones and disadvantaged spectators in order to provide them high definition images.

An important factor that determines the quality of the images is the time in which the drones start to film the events. The function defining the viewer satisfaction is depicted in the Figure 1.

The total satisfaction of the viewer, named $v s(x)$ where $x$ represents a solution, is obtained by averaging the sum of the satisfaction experienced from each action on the whole event.
The satisfaction is maximized if the filming drone arrives at the action location and the supporting drone at the location from where it can relay before $t_{\text {start }}$, and it decreases linearly until becoming 0 when $t=t_{\text {stop }}$. The definition of viewer satisfaction matches with the definition of Soft Time Window, because the only effect of a drone not arriving before $t_{\text {start }}$ at the action location is to reduce the overall satisfaction of the viewer without invalidating the solution of the problem.

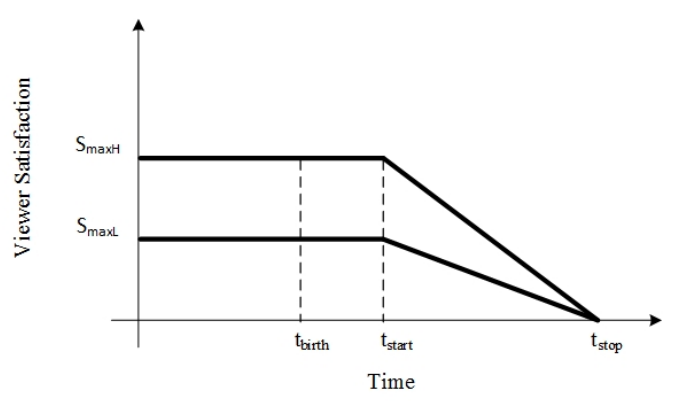

Figure 1. Game action's soft time window for the viewer's satisfaction.

Given a solution $x$, let $d(x)$ be the total distance travelled by the filming and the supporting drones. The bi-objective formulation of the problem is reported in the following

$$
\mathrm{SEF}-\mathrm{SD}=\{\max v s(x), \min d(x): x \in X\},
$$

where the set $X$ represents the feasible region. More details on the mathematical formulation and the definition of set $X$ are reported in the Appendix.

A solution to problem (1) is represented by the set of efficient solutions. Given an efficient solution $\bar{x}$, the vector $[v s(\bar{x}), d(\bar{x})]$ is the so-called Pareto point in the criteria space associated with the efficient solution $\bar{x}$. The set of efficient solutions $\bar{X}$ contains the non-dominated Pareto points.

Given two efficient solutions $\bar{x}_{1}$ and $\bar{x}_{2}$, we say that solution $\bar{x}_{1}$ dominates $\bar{x}_{2}$ if $v s\left(\bar{x}_{1}\right) \geq v s\left(\bar{x}_{2}\right), d\left(\bar{x}_{1}\right) \leq$ $d\left(\bar{x}_{2}\right)$, and at least one inequality is strict.

We can distinguish two typologies of non-dominated solutions, the first, named supported non-dominated, lies on the convex hull of the Pareto front, the second ones are known as non-supported non-dominated solutions.

b) Solution approaches: In this paper we propose to solve problem (1) by using three different methods. The first two are based on scalarizing function $S(\cdot, x)$. In particular, we consider a convex combination with $S(\lambda, x)=\lambda \alpha d(x)-(1-\lambda) v s(x)$ to be minimized and $\lambda \in[0,1]$. Varying the parameter $\lambda$, different supported non-dominated solutions are obtained. The term $\alpha$ is used to scale the function $d(x)$. In particular, $\alpha=1 /\left(d^{N}-d^{U}\right)$ 
where $d^{N}$ is the nadir point and $d^{U}$ is the utopia point associated with the function $d(x)$. The problem is defined as $\mathrm{SEF}-\mathrm{SD}^{\lambda}=\{\min S(\lambda, x): x \in X\}$.

The second scalarizing technique uses the reference point methodology. A reference point is a vector $\bar{q}$ containing values for $d$ and $v s$ that the decision maker would to achieve. In other words, $\bar{q}$ represents the decision maker's desiderata. In this case, $S(\bar{q}, y, x)=\min \left\{y_{1}\left(\bar{q}_{1}-d(x)\right), y_{2}\left(v s(x)-\bar{q}_{2}\right)\right\}+$ $\gamma\left[y_{1}\left(\bar{q}_{1}-d(x)\right)+y_{2}\left(v s(x)-\bar{q}_{2}\right)\right], \gamma<<1$. Maximizing $S(\bar{q}, y, x)$ means to obtain a Pareto optimal solution $[d(\bar{x}), v s(\bar{x})]$ that is the nearest, with respect to the Chebishev norm, to the reference point $\bar{q}$. The problem to be solved is $\mathrm{SEF}-\mathrm{SD}^{\bar{q}}=\{\max S(\bar{q}, y, x): x \in X\}$.

The last technique refers to the so-called $\epsilon$-constraint method. The main idea of this strategy is to solve a singleobjective problem optimizing a function and treats the other one as a hard constraint. In our application, we decide to maximize the viewer satisfaction imposing a limitation $\epsilon$ on the distance travelled. Varying the parameter $\epsilon$, different solution are determined. The problem addressed with the $\epsilon$-constraint method is denoted as SEF$\mathrm{SD}^{\epsilon}=\{\max v s(x): x \in X, d(x) \leq \epsilon\}$.

\section{ILLUSTRATIVE EXAMPLE}

In order to show how the considered solution strategies behave in solving the SEF-SD, we present in this section an example reporting the Pareto optimal solutions obtained by solving SEF-SD ${ }^{\lambda}, \mathrm{SEF}_{-} \mathrm{SD}^{\bar{q}}$, and SEF-SD ${ }^{\epsilon}$. The three models are solved with Cplex and implemented in Lingo [10].

We generate a simple example, whose main characteristics are described in what follows. We consider two filming drones and two supporting ones. The events to be filmed are randomly distributed in the game field and we consider 5 events, three of them are located in the 1-Hop area. There is one initial position, where both filming and supporting drones start their work and one final position. Two positions are devoted to the supporting drones.

All the Pareto solutions for the considered instance are depicted in Figure 2.

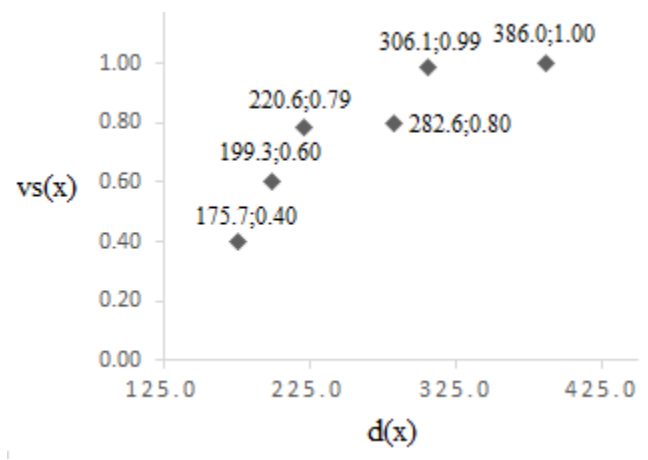

Figure 2. Pareto optimal solutions for the considered example.
It is worth observing that five solutions represent supported non-dominated points, whereas the Pareto point $[282.60 ; 0.80]$ is associated with a non-supported nondominated solution.

Table I reports the Pareto solutions obtained by solving

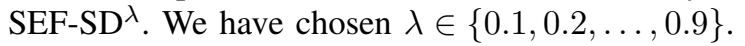

Table I. PARETo OPTIMAL SOLUTIONS DETERMINED BY SOLVING

\begin{tabular}{r|cr|}
\multicolumn{2}{|c|}{ SEF-SD $^{\lambda}$} \\
\multicolumn{1}{c|}{ PO } \\
\hline 0.1 & $3(x)$ & $v s(x)$ \\
\hline $0.2-0.3$ & 306.0 & 1.00 \\
$0.4-0.6$ & 220.6 & 0.99 \\
$0.7-0.9$ & 175.7 & 0.49 \\
\hline
\end{tabular}

Column $\lambda$ reports the values of $\lambda$ for which SEF-SD ${ }^{\lambda}$ returns the corresponding solution. Column PO shows the Pareto optimal solutions obtained. It is evident that for $\lambda=0.1$, the corresponding $d(x)$ represents the nadir point for function $d$, whereas when $\lambda$ is set equal to 0.9 , the values for $d(x)$ of the corresponding Pareto optimal solution is the utopia point. An expected results is that ${\mathrm{SEF}-\mathrm{SD}^{\lambda}}^{\lambda}$ is not able to determine the non-supported non-dominated solution, i.e. [288.1,0.80]. This is true in general since optimizing the convex combination of the objective functions leads to search solutions in the corner of the convex hull.

The Pareto optimal solutions obtained with SEF-SD ${ }^{\epsilon}$ with $\epsilon \in\{400,300,200,100\}$ are given in Table II.

Table II. PARETO OPTIMAL SOLUTIONS DETERMINED BY SOLVING

\begin{tabular}{c|cr|}
\multicolumn{3}{|c|}{ SEF-SD $^{\epsilon}$} \\
$\epsilon$ & \multicolumn{2}{|c|}{ PO } \\
& $d(x)$ & vs $(x)$ \\
\hline 400 & 386.0 & 1.00 \\
300 & 288.1 & 0.80 \\
200 & 199.3 & 0.60 \\
100 & \multicolumn{2}{|c|}{ infeasible } \\
\hline
\end{tabular}

Problem SEF-SD ${ }^{\epsilon}$ is strongly affected by the parameter $\epsilon$. Indeed, table II shows that only three Pareto optimal solutions are determined considering the chosen values for $\epsilon$. However, SEF-SD ${ }^{\epsilon}$ overcomes the issue related to the determination of non-supported non-dominated solutions observed for SEF-SD ${ }^{\lambda}$. Indeed, for $\epsilon=300, \mathrm{SEF}_{-} \mathrm{SD}^{\epsilon}$ returns the Pareto solution [288.1,0.80].

The Pareto optimal solutions determined by SEF-SD ${ }^{\bar{q}}$ are given in table III.

We solve SEF-SD ${ }^{\bar{q}}$ considering $\bar{q}_{1} \in$ $\{400,300,200,100\}$ and $\bar{q}_{2} \in\{0,0.2, \ldots, 0.8,1\}$. Column $\bar{q}$ of table III reports the reference points for which the corresponding Pareto optimal solutions are obtained. Table III shows the potential of SEF-SD ${ }^{\bar{q}}$ that is able to determine all the Pareto optimal solutions. However, the number of times SEF-SD ${ }^{\bar{q}}$ is solved is higher that the other two methods. On the other hand, SEF-SD ${ }^{\bar{q}}$ takes into account the decision maker's desiderata providing solutions near to his/her aspiration. 
Table III. PAReto OPTIMAL SOLUTIONS DETERMined BY SOLVING $\mathrm{SEF}-\mathrm{SD}^{\bar{q}}$

\begin{tabular}{l|cr|}
\multicolumn{1}{c|}{$\bar{q}$} & \multicolumn{2}{|c}{ PO } \\
& $d(x)$ & $v s(x)$ \\
\hline$[400,1]$ & 386.0 & 1.00 \\
{$[300,1],[400,0.8],[400,0.6]$} & 306.1 & 0.99 \\
{$[400,0.4]$} & & \\
{$[300,0.8]$} & 282.6 & 0.80 \\
{$[200,1],[200,0.8],[300,0.6]$} & 220.6 & 0.79 \\
{$[300,0.4],[400,0.2],[400,0]$} & & \\
{$[100,1],[200,0.6],[200,0.4]$} & 199.3 & 0.60 \\
{$[300,0.2],[300,0]$} & & \\
{$[100,0.8],[100,0.6],[100,0.4]$} & 175.7 & 0.40 \\
{$[100,0.2],[100,0],[200,0.2]$} & & \\
{$[200,0]$} & & \\
\hline
\end{tabular}

\section{CONCLusions}

In this paper we address the problem of filming sport events with drones. The aim is to provide high quality images to the spectators within the stadium. The images sent from the corners of the stadium to the spectators located to the opposite ones are of low quality. In order to ensure a maximum viewer satisfaction, supporting drones are considered and they play the role of relay between the filming drones and the disadvantaged spectators.

Beside the quality of the images, the distance travelled by the drones is taken into account. The two objectives are in conflict with each other, thus we formulate the problem as a bi-objective mixed integer program. Three methods are applied to solve the problem. Two of them are based on scalarizing functions and the other on the $\epsilon$-constraint strategy. The behavior of the proposed approaches are shown considering an illustrative example.

\section{APPENDIX - VARIABLES AND MODEL ASSUMPTIONS}

In this section we introduce all the parameters, variables, constraints and assumptions used to formulate the proposed mathematical model.

\section{Parameters}

- $F_{\text {stadium }}=B_{s} \times H_{s}$ size-limited stadium;

- $F_{\text {field }}=B_{f} \times H_{f}$ size-limited field;

- $0 \rightarrow T$ event time duration;

- $M=\{1, \ldots, m\}$ set of $m$ drones able to move in 2 dimensions;

- $\bar{M}=\{1, \ldots, \bar{m}\}$ set of $\bar{m}$ drones supporting the drones in $M$;

- $N=\{1 \ldots n\}$ actions spatially distributed in $F$ and timedistributed in $0 \rightarrow T, n$ represents the final event;

- $S=\{1, \ldots, p\}$ set of the supporting positions;

- $\tilde{N}=\{1, \ldots, m\}$ set of the initial positions of the drones, before they start moving to film/support the actions;

- $t_{\text {birth }, i}$ birth time of action $i, \forall i \in N \backslash\left\{N_{1} \ldots N_{m}\right\}$;

- $t_{\text {start }, i}$ start time of action $i, \forall i \in N \backslash\left\{N_{1} \ldots N_{m}\right\}$;

- $t_{\text {stop }, i}$ stop time of action $i, \forall i \in N \backslash\left\{N_{1} \ldots N_{m}\right\}$;

- $t_{\text {birth }, i}<t_{\text {start }, i}<t_{\text {stop }, i} \forall i \in N \backslash\left\{N_{1} \ldots N_{m}\right\}$;

- $t_{\text {stop }, i}<t_{\text {birth }, j} \forall i, j \in N \backslash\left\{N_{1} \ldots N_{m}\right\}$ with $i<j$ ensures that actions are sequential and non-simultaneous;

- $t_{s t o p, n}=T$ ensures that all actions terminate within the given time frame.

- $d_{i j}$ Euclidean distance between the location of action (supporting position) $i$ and action (supporting position) $j$;

- $d_{i k} \leq d_{i j}+d_{j k}$ triangle inequality for the distances among filming/supporting actions locations $\forall i, j, k \in N$;
- $V S_{\max }=V S_{\max H D}=1$ maximum satisfaction obtainable by the farthest viewer in a single action when both the drones are well placed;

- $V S_{\max S D}=1 / 2$ maximum satisfaction obtainable by the farthest viewer in a single action when no support is guaranteed to the filming drone;

\section{Variables}

- $t_{a r r, i}^{k}$ arrival time of filming drone $k$ to the location of action $i \forall i \in N \backslash\left\{N_{1} \ldots N_{m}\right\}$ and $\forall k \in M$;

- $t_{a r r, p}^{k}$ arrival time of supporting drone $k$ to the location of supporting position $p, \forall p \in S$ and $\forall k \in \bar{M}$;

- $t_{d e p, i}^{k}$ departure time of filming drone $k$ from the location of action $i \forall i \in N \backslash\left\{N_{n}\right\}$ and $\forall k \in M$;

- $t_{d e p, p}^{k}$ departure time of supporting drone $k$ from the location of supporting action $p, \forall p \in S$ and $\forall k \in \bar{M}$;

- $t_{i \rightarrow j}^{k}=\frac{d_{i j}}{v}$ time required by drone $k$ to move from filming/supporting action $i$ to filming/supporting action $j$ $\forall i, j \in N$ and $\forall k \in M \cup \bar{M}$;

- $x_{i j}^{k}$ on/off variable with the following meaning:

$$
x_{i j}^{k}= \begin{cases}1 & \text { if arc } i-j \text { is crossed by } \\ & \text { filming/supporting drone } k \in M \cup \bar{M} \\ 0 & \text { otherwise }\end{cases}
$$

- $y_{i}^{k}$ on/off variable with the following meaning:

$$
y_{i}^{k}= \begin{cases}1 & \begin{array}{l}
\text { if filming drone } k \in M \text { was on } \\
\text { the position } i \text { of game action }
\end{array} \\
0 & \text { otherwise }\end{cases}
$$

- $V S_{i, H D}^{k}$ viewer's satisfaction due to the drone $k$ that filmed action $i$ when the action belongs to the set 1-HOP;

- $V S_{i, S D}^{k}$ viewer's satisfaction due to the drone $k$ that filmed action $i$ when the action does not belong to the set 1-HOP.

\section{Assumptions}

- The time and spatial sequences of actions are known;

- Each action (except the last one) must be filmed by exactly one drone;

- Since the last game action represents a dummy location, where the drones come together, so that maintenance operations can be performed, the distance traveled to reach this final position is not considered in evaluating the total distance travelled by the drones.

- All actions $i \in N$ have their own Soft Time Window already presented in Figure 1;

- All actions $i \in N$ have a set of supporting positions, which are good for relaying the transmission. We assume that $s_{i p}=1$ if position $p \in P$ is able to support the event $i \in N, s_{i p}=0$, otherwise. When the position of the action is in the 1-Hop zone, then there is no needs of using supporting drones.

\section{Problem Formulation}

The objective functions are the total distance traveled by the drones involved in the sport event filming (to be minimized) and the viewer satisfaction to be maximized. This can be expressed as

$$
\begin{array}{ll}
\min & \sum_{k \in M} \sum_{i \in N \cup S} \sum_{j \in N \cup S} d_{i j} \cdot x_{i j}^{k} \\
\max & \frac{\sum_{i \in N} \sum_{k \in M}\left(V S_{i, H D}^{k}+V S_{i, S D}^{k}\right)}{n}
\end{array}
$$


Thise objectives have to be accomplished by satisfying the constraints reported in the following.

Each action have to be filmed by exactly one drone.

$$
\begin{gathered}
\sum_{k \in M} y_{i}^{k}=1 \quad \forall i \in N \backslash\{n\} \\
\sum_{k \in M} y_{n}^{k}=m
\end{gathered}
$$

A supporting drone positioned in $p$ could support the drone, which films the actions $i \notin 1$-HOP. We define the binary variables $\beta_{i, p}^{k}$ that is set equal to 1 when a supporting drone $k \in \bar{M}$ is in position $p$ for supporting the action $i$.

$$
\begin{gathered}
\beta_{i, p}^{k} \leq s_{i, p} \forall i \in N \backslash 1-\text { Hop }, p \in S, k \in \bar{M} \\
\sum_{k \in \bar{M}} \sum_{p \in S} \beta_{i, p}^{k} \leq 1 \quad \forall i \in N \backslash 1-\text { Hop }
\end{gathered}
$$

The binary variable $y_{i}^{k}$ is equal to 1 when the drone $k \in M$ films action $i$.

$$
\begin{aligned}
& y_{i}^{k} \geq x_{j i}^{k} \quad \forall i, j \in N \text { and } \forall k \in M \\
& y_{i}^{k} \geq x_{i j}^{k} \quad \forall i, j \in N \text { and } \forall k \in M
\end{aligned}
$$

Each drone starts from its initial position and all drones end at the final action $n$.

$$
\begin{gathered}
\sum_{i \in \tilde{N}} \sum_{j \in N} x_{i j}^{k}=1 \quad \forall k \in M \\
\sum_{i \in\{N-\{n\}\}} x_{i n}^{k}=1 \quad \forall k \in M
\end{gathered}
$$

Flow constraints defining the path of the filming drone $k \in$ $M$.

$$
\begin{gathered}
\sum_{k \in M} \sum_{j \in\{\tilde{N} \cup N-\{n\}\}} x_{j i}^{k}=1 \quad \forall i \in N \\
\sum_{k \in M} \sum_{j \in N} x_{i j}^{k}=1 \quad \forall i \in N-\{n\} \\
\sum_{i \in N \cup \tilde{N}} x_{i z}^{k}-\sum_{j \in N \cup \tilde{N}} x_{z j}^{k}=0 \\
\forall z \in N-\{n\} \text { and } \forall k \in M
\end{gathered}
$$

Flow constraints defining the path of the supporting drone $k \in \bar{M}$.

$$
\begin{gathered}
\sum_{i \in \tilde{N}} \sum_{j \in S} x_{i j}^{k} \leq 1, \quad \forall k \in \bar{M} . \\
\sum_{i \in \tilde{N} \cup S} x_{i n}^{k} \leq 1, \quad \forall k \in \bar{M} . \\
\sum_{i \in S \cup \tilde{N}} x_{i z}^{k}-\sum_{j \in S \cup\{n\}} x_{z j}^{k}=0, \quad \forall k \in \bar{M}, z \in S .
\end{gathered}
$$

The supporting point $p \in S$ have to be visited by exactly one supporting drone.

$$
\sum_{k \in \bar{M}} x_{i j}^{k} \leq 1, \quad \forall i \in S \cup \tilde{N}, j \in S \cup\{n\} .
$$

All drones start at time 0 , that is, $t_{a r r, i}^{k}=0, \forall i \in \tilde{N}$ and $\forall k \in$ $M \cup \bar{M}$.
The arrival time to action $j$ of drone $k$ is equal to the departure time from node $i$ plus the time needed to reach node $j$.

$$
\begin{gathered}
t_{a r r, j}^{k}=\sum_{i \in N \cup S \cup \tilde{N}-\{n\}}\left(t_{d e p, i}^{k}+t_{i \rightarrow j}^{k}\right) x_{i j}^{k} \\
\forall j \in N \cup S \cup \tilde{N} \text { and } \forall k \in M \cup \bar{M}
\end{gathered}
$$

A drone can leave an action after the action is concluded and before the end of the match.

$$
\begin{aligned}
& t_{\text {dep }, i}^{k} \geq t_{\text {stop }, i} \cdot y_{i}^{k}, \forall i \in N \text { and } \forall k \in M \\
& t_{\text {dep }, i}^{k} \leq t_{\text {stop }, n} \cdot y_{i}^{k}, \forall i \in N \text { and } \forall k \in M
\end{aligned}
$$

$t_{d e p, p}^{k} \geq t_{s t o p, i} \cdot \beta_{i, p}^{k}, \forall i \in N$ and $\forall p \in S$ and $\forall k \in \bar{M}$

$t_{d e p, p}^{k} \leq t_{s t o p, n} \cdot \beta_{i, p}^{k}, \forall i \in N$ and $\forall p \in S$ and $\forall k \in \bar{M}$

The following constraints are introduced in order to linearize the satisfaction function.

$$
\begin{aligned}
& \delta_{1, i}^{k}+\delta_{2, i}^{k}+\delta_{3, i}^{k}=y_{i}^{k}, \forall i \in N \text { and } \forall k \in M \\
& 0 \leq \zeta_{1, i}^{k} \leq t_{\text {start }, i} \delta_{1, i}^{k}, \forall i \in N \text { and } \forall k \in M
\end{aligned}
$$

$0 \leq \zeta_{2, i}^{k} \leq\left(t_{\text {stop }, i}-t_{\text {start }, i}\right) \delta_{2, i}^{k}, \forall i \in N$ and $\forall k \in M$

$0 \leq \zeta_{3, i}^{k} \leq\left(t_{\text {stop }, n}-t_{\text {stop }, i}\right) \delta_{3, i}^{k}, \forall i \in N$ and $\forall k \in M$

$$
t_{\text {arr }, i}^{k}=t_{\text {start }, i} \delta_{2, i}^{k}+t_{\text {stop }, i} \delta_{3, i}^{k}+\zeta_{1, i}^{k}+\zeta_{2, i}^{k}+\zeta_{3, i}^{k},
$$

Definition of $V S_{i, H D}^{k}$.

$$
\begin{gathered}
V S_{i, H D}^{k}=V S_{\max H D} \delta_{1, i}^{k}+V S_{\max H D} \delta_{2, i}^{k}+ \\
+\left(\frac{-V S_{\max H D}}{t_{\text {stop }, i}-t_{\text {start }, i}}\right) \zeta_{2, i}^{k}, \forall i \in 1 \text {-Hop and } \forall k \in M .
\end{gathered}
$$

The following constraints allow to define the satisfaction for the actions $i \notin 1-\mathrm{HOP}$.

The binary variables $\alpha_{i p}^{k}$ is equal to 1 if the supporting drone $k \in \bar{M}$ starts to support action $i$ before the starting time of action $i$.

$$
\begin{aligned}
& t_{a r r, i}^{k}-t_{a r r, p}^{\tilde{k}}+\Theta\left(1-y_{i}^{k}\right) \geq \Theta\left(\alpha_{i, p}^{\tilde{k}}-1\right) \\
& \forall i \in N \backslash 1 \text {-Hop, } \forall k \in M, \tilde{k} \in \bar{M} \quad \forall p \in S
\end{aligned}
$$

The binary variables $l_{i, p}^{\tilde{k}}$ define whether the supporting drone $k \in \bar{M}$ support the action $i$, i.e. $\beta_{i, p}^{\tilde{k}}=1$ and they arrive before the actions start $\left(\alpha_{i, p}^{\tilde{k}}=1\right)$.

$l_{i, p}^{\tilde{k}} \geq \alpha_{i, p}^{\tilde{k}}+\beta_{i, p}^{\tilde{k}}-1, \forall i \in N \backslash 1$-Hop, $\forall \tilde{k} \in \bar{M} \quad \forall p \in S$

$l_{i, p}^{\tilde{k}} \leq \frac{1}{2}\left(\alpha_{i, p}^{\tilde{k}}+\beta_{i, p}^{\tilde{k}}\right), \forall i \in N \backslash 1$-Hop, $\forall \tilde{k} \in \bar{M} \quad \forall p \in S$ 
In the case $l_{i, p}^{\tilde{k}}=1$, we have an HD satisfaction. The following constraints allow to define the HD satisfaction for the actions $i \notin 1-\mathrm{HOP}$.

$$
\begin{aligned}
& \bar{\delta}_{1 i}^{k} \geq \sum_{\tilde{k} \in \bar{M}} \sum_{p \in S} l_{i, p}^{\tilde{k}}+\delta_{1 i}^{k}-1, \forall i \in N \backslash 1 \text {-Hop, } \forall k \in M \\
& \bar{\delta}_{1 i}^{k} \leq \frac{1}{2}\left(\sum_{\tilde{k} \in \bar{M}} \sum_{p \in S} l_{i, p}^{\tilde{k}}+\delta_{1 i}^{k}\right), \forall i \in N \backslash 1 \text {-Hop, } \forall k \in M \\
& \bar{\delta}_{2 i}^{k} \geq \sum_{\tilde{k} \in \bar{M}} \sum_{p \in S} l_{i, p}^{\tilde{k}}+\delta_{2 i}^{k}-1, \forall i \in N \backslash 1 \text {-Hop, } \forall k \in M \\
& \bar{\delta}_{2 i}^{k} \leq \frac{1}{2}\left(\sum_{\tilde{k} \in \bar{M}} \sum_{p \in S} l_{i, p}^{\tilde{k}}+\delta_{2 i}^{k}\right), \forall i \in N \backslash 1 \text {-Hop }, \forall k \in M \\
& \bar{\zeta}_{2, i}^{k} \geq \sum_{\tilde{k} \in \bar{M}} \sum_{p \in S} l_{i, p}^{\tilde{k}}+\zeta_{2 i}^{k}-1, \forall i \in N \backslash 1 \text {-Hop, } \forall k \in M \\
& \bar{\zeta}_{2 i}^{k} \leq \frac{1}{2}\left(\sum_{\tilde{k} \in \bar{M}} \sum_{p \in S} l_{i, p}^{\tilde{k}}+\zeta_{2 i}^{k}\right), \forall i \in N \backslash 1 \text {-Hop }, \forall k \in M
\end{aligned}
$$

When $l_{i, p}^{\tilde{k}}=0, \forall \tilde{k} \in \bar{M}$, then no supporting drone is available for the action $i$. In this case, we have an SD satisfaction. The following constraints are used to define the satisfaction for the actions $i \notin 1-\mathrm{HOP}$.

$$
\begin{aligned}
& f_{1 i}^{k} \geq-\sum_{\tilde{k} \in \bar{M}} \sum_{p \in S} l_{i, p}^{\tilde{k}}+\delta_{1 i}^{k}, \forall i \in N \backslash 1 \text {-Hop, } \forall k \in M \\
& f_{1 i}^{k} \leq \frac{1}{2}\left(1-\sum_{\tilde{k} \in \bar{M}} \sum_{p \in S} l_{i, p}^{\tilde{k}}+\delta_{1 i}^{k}\right), \forall i \in N \backslash 1 \text {-Hop, } \forall k \in M \\
& f_{2 i}^{k} \geq-\sum_{\tilde{k} \in \bar{M}} \sum_{p \in S} l_{i, p}^{\tilde{k}}+\delta_{2 i}^{k}, \forall i \in N \backslash 1 \text {-Hop, } \forall k \in M \\
& f_{2 i}^{k} \leq \frac{1}{2}\left(1-\sum_{\tilde{k} \in \bar{M}} \sum_{p \in S} l_{i, p}^{\tilde{k}}+\delta_{2 i}^{k}\right), \forall i \in N \backslash 1 \text {-Hop, } \forall k \in M \\
& f_{3, i}^{k} \geq-\sum_{\tilde{k} \in \bar{M}} \sum_{p \in S} l_{i, p}^{\tilde{k}}+\zeta_{2 i}^{k}, \forall i \in N \backslash 1 \text {-Hop, } \forall k \in M, \\
& f_{3 i}^{k} \leq \frac{1}{2}\left(1-\sum_{\tilde{k} \in \bar{M}} \sum_{p \in S} l_{i, p}^{\tilde{k}}+\zeta_{2 i}^{k}\right), \forall i \in N \backslash 1 \text {-Hop, } \forall k \in M
\end{aligned}
$$

The satisfaction level of the spectators for the actions $i \notin$ 1 -HOP is defined in equation (45).

$$
\begin{gathered}
V S_{i, S D}^{k}=V S_{\max H D} \bar{\delta}_{1, i}^{k}+V S_{\max H D} \bar{\delta}_{2, i}^{k}+ \\
\left(\frac{-V S_{\max H D}}{t_{\text {stop }, i}-t_{\text {start }, i}}\right) \bar{\zeta}_{2, i}^{k}+ \\
+V S_{\max S D} f_{1, i}^{k}+V S_{\max S D} f_{2, i}^{k}+ \\
+\left(\frac{-V S_{\text {max } S D}}{t_{\text {stop }, i}-t_{\text {start }, i}}\right) f_{3, i}^{k}
\end{gathered}
$$

\section{REFERENCES}

[1] E. Natalizio, R. Surace, V. Loscrí, F. Guerriero, and T. Melodia, "Two families of algorithms to film sport events with flying robots," in 2013 IEEE 10th International Conference on Mobile Ad-Hoc and Sensor Systems, Oct 2013, pp. 319-323.

[2] R. Baldacci, A. Mingozzi, and R. Roberti, "Recent exact algorithms for solving the vehicle routing problem under capacity and time window constraints," European Journal of Operational Research, vol. 218, pp. 1-6, 2012.

[3] F. Z. Qureshi and D. Terzopoulos, "Surveillance in virtual reality: system design and multi-camera control," Proc. of IEEE Int. Conf. on Computer Vision and Pattern Recognition, CVRP 07, pp. 1-8, June 2007.

[4] K. S. Abdallah and J. Jang, "An exact solution for vehicle routing problems with semi-hard resource constraints," Computers \& Industrial Engineering, vol. 76, pp. 366-377, 2014.

[5] H. I. Calvete, C. Galé, M. Oliveros, and B. Sánchez-Valverde, "A goal programming approach to vehicle routing problems with soft time windows," European Journal of Operational Research, vol. 177, pp. 1720-1733, 2007.

[6] A. Wierzbicki, "Basic properties of scalarizing functionals for multiobjective optimization," Mathematische Operationsforschung und Statistik, s. Optimization, vol. 8, pp. 55-60, 1977.

[7] _ , "On the completeness and constructiveness of parametric characterizations to vector optimization problems," OR Spektrum, vol. 8, pp. 73-87, 1986.

[8] J. F. Bérubé, M. Gendreau, and J. Y. Potvin, "An exact $\epsilon$-constraint method for bi-objective combinatorial optimization problems: Application to the traveling salesman problem with profits," European Journal of Operational Research, vol. 194, pp. 39-50, 2009.

[9] E. Yanmaz, S. Hayat, J. Scherer, and C. Bettstetter, "Experimental performance analysis of two-hop aerial 802.11 networks," in 2014 IEEE Wireless Communications and Networking Conference (WCNC). IEEE, 2014, pp. 3118-3123.

[10] L. Schrage, "Optimization modeling with lingo. lindo systems," Inc., Chicago, IL, 2006.

$\forall i \in N \backslash 1$-Hop and $\forall k \in M$ 